Korean J. Math. 19 (2011), No. 2, pp. 149-161

\title{
STABILITY OF THE JENSEN TYPE FUNCTIONAL EQUATION IN BANACH ALGEBRAS: A FIXED POINT APPROACH
}

\author{
Choonkil Park, Won-Gil Park*, Jung Rye Lee And \\ Themistocles M. Rassias
}

\begin{abstract}
Using fixed point methods, we prove the generalized Hyers-Ulam stability of homomorphisms in Banach algebras and of derivations on Banach algebras for the following Jensen type functional equation:
\end{abstract}

$$
f\left(\frac{x+y}{2}\right)+f\left(\frac{x-y}{2}\right)=f(x) .
$$

\section{Introduction and preliminaries}

The stability problem of functional equations was originated from a question of Ulam [30] concerning the stability of group homomorphisms: Let $\left(G_{1}, \star\right)$ be a group and let $\left(G_{2}, \diamond, d\right)$ be a metric group with the metric $d(\cdot, \cdot)$. Given $\varepsilon>0$, does there exist a $\delta(\varepsilon)>0$ such that if a mapping $h: G_{1} \rightarrow G_{2}$ satisfies the inequality

$$
d(h(x \star y), h(x) \diamond h(y))<\delta
$$

for all $x, y \in G_{1}$, then there is a homomorphism $H: G_{1} \rightarrow G_{2}$ with

$$
d(h(x), H(x))<\varepsilon
$$

Received February 9, 2011. Revised June 7, 2011. Accepted June 10, 2011. 2000 Mathematics Subject Classification: 39A10, 39B72, 47H10, 46B03.

Key words and phrases: Jensen type functional equation, fixed point, homomorphism in Banach algebra, generalized Hyers-Ulam stability, derivation on Banach algebra.

The third author was supported by Basic Science Research Program through the National Research Foundation of Korea funded by the Ministry of Education, Science and Technology (NRF-2010-0009232).

${ }^{*}$ Corresponding author. 
for all $x \in G_{1}$ ? If the answer is affirmative, we would say that the equation of homomorphism $H(x \star y)=H(x) \diamond H(y)$ is stable. The concept of stability for a functional equation arises when we replace the functional equation by an inequality which acts as a perturbation of the equation. Thus the stability question of functional equations is that how do the solutions of the inequality differ from those of the given functional equation?

Hyers [8] gave a first affirmative partial answer to the question of Ulam for Banach spaces. Let $X$ and $Y$ be Banach spaces. Assume that $f: X \rightarrow Y$ satisfies

$$
\|f(x+y)-f(x)-f(y)\| \leq \varepsilon
$$

for all $x, y \in X$ and some $\varepsilon \geq 0$. Then there exists a unique additive mapping $T: X \rightarrow Y$ such that

$$
\|f(x)-T(x)\| \leq \varepsilon
$$

for all $x \in X$.

Th.M. Rassias [20] provided a generalization of Hyers' Theorem which allows the Cauchy difference to be unbounded.

Theorem 1.1. (Th.M. Rassias). Let $f: E \rightarrow E^{\prime}$ be a mapping from a normed vector space $E$ into a Banach space $E^{\prime}$ subject to the inequality

$$
\|f(x+y)-f(x)-f(y)\| \leq \varepsilon\left(\|x\|^{p}+\|y\|^{p}\right)
$$

for all $x, y \in E$, where $\varepsilon$ and $p$ are constants with $\varepsilon>0$ and $p<1$. Then the limit

$$
L(x)=\lim _{n \rightarrow \infty} \frac{f\left(2^{n} x\right)}{2^{n}}
$$

exists for all $x \in E$ and $L: E \rightarrow E^{\prime}$ is the unique additive mapping which satisfies

$$
\|f(x)-L(x)\| \leq \frac{2 \varepsilon}{2-2^{p}}\|x\|^{p}
$$

for all $x \in E$. Also, if for each $x \in E$ the mapping $f(t x)$ is continuous in $t \in \mathbb{R}$, then $L$ is $\mathbb{R}$-linear.

The above inequality (1.1) that was introduced for the first time by Th.M. Rassias [20] for the proof of the stability of the linear mapping between Banach spaces has provided a lot of influence in the development of what is now known as generalized Hyers-Ulam stability or as Hyers-Ulam-Rassias stability of functional equations. Beginning around the year 1980 the topic of approximate homomorphisms, or the stability 
of the equation of homomorphism, was studied by a number of mathematicians. Găvruta [7] extended the Hyers-Ulam stability by proving the following theorem in the spirit of Th.M. Rassias' approach.

TheOREm 1.2. [7] Let $f: E \rightarrow E^{\prime}$ be a mapping for which there exists a function $\varphi: E \times E^{\prime} \rightarrow[0, \infty)$ such that

$$
\begin{aligned}
\widetilde{\varphi}(x, y) & :=\sum_{j=0}^{\infty} 2^{-j} \varphi\left(2^{j} x, 2^{j} y\right) \\
\|f(x+y)-f(x)-f(y)\| & \leq \varphi(x, y)
\end{aligned}
$$

for all $x, y \in E$. Then there exists a unique additive mapping $T: E \rightarrow E^{\prime}$ such that

$$
\|f(x)-T(x)\| \leq \frac{1}{2} \widetilde{\varphi}(x, x)
$$

for all $x \in E$.

The stability problems of several functional equations have been extensively investigated by a number of authors and there are many interesting results concerning this problem (see $[1,2,4,5,10,11,13,14,15$, $16,17,18,19,21,22,23,24,25,26,27,28,29])$.

We recall the following theorem by Diaz and Margolis. The reader is referred to the book of D.H. Hyers, G. Isac and Th.M. Rassias [9] for an extensive account of fixed point theory with several applications.

Theorem 1.3. [6] Let $(X, d)$ be a complete generalized metric space and let $J: X \rightarrow X$ be a strictly contractive mapping with Lipschitz constant $L<1$. Then for each given element $x \in X$, either

$$
d\left(J^{n} x, J^{n+1} x\right)=\infty
$$

for all nonnegative integers $n$ or there exists a positive integer $n_{0}$ such that

(1) $d\left(J^{n} x, J^{n+1} x\right)<\infty$ for all $n \geq n_{0}$;

(2) the sequence $\left\{J^{n} x\right\}$ converges to a fixed point $y^{*}$ of $J$;

(3) $y^{*}$ is the unique fixed point of $J$ in the set $Y=\{y \in X \mid$ $\left.d\left(J^{n_{0}} x, y\right)<\infty\right\}$;

(4) $d\left(y, y^{*}\right) \leq \frac{1}{1-L} d(y, J y)$ for all $y \in Y$.

This paper is organized as follows: In Section 2, using the fixed point method, we prove the generalized Hyers-Ulam stability of homomorphisms in Banach algebras for the Jensen type functional equation. 
In Section 3, using the fixed point method, we prove the generalized Hyers-Ulam stability of derivations on Banach algebras for the Jensen type functional equation.

In 1996, G. Isac and Th.M. Rassias [12] were the first to provide applications of stability theory of functional equations for the proof of new fixed point theorems with applications.

Throughout this paper, assume that $A$ is a real Banach algebra with norm $\|\cdot\|_{A}$ and that $B$ is a real Banach algebra with norm $\|\cdot\|_{B}$.

\section{Stability of homomorphisms in Banach algebras}

For a given mapping $f: A \rightarrow B$, we define

$$
D f(x, y):=f\left(\frac{x+y}{2}\right)+f\left(\frac{x-y}{2}\right)-f(x)
$$

for all $x, y \in A$.

Note that an $\mathbb{R}$-linear mapping $H: A \rightarrow B$ is called a homomorphism in Banach algebras if $H$ satisfies $H(x y)=H(x) H(y)$ for all $x, y \in A$.

Let $X$ be a set. A function $d: X \times X \rightarrow[0, \infty]$ is called a generalized metric on $X$ if $d$ satisfies

(1) $d(x, y)=0$ if and only if $x=y$;

(2) $d(x, y)=d(y, x)$ for all $x, y \in X$;

(3) $d(x, z) \leq d(x, y)+d(y, z)$ for all $x, y, z \in X$.

We prove the generalized Hyers-Ulam stability of homomorphisms in Banach algebras for the functional equation $D f(x, y)=0$.

Theorem 2.1. Let $f: A \rightarrow B$ be a mapping for which there exists a function $\varphi: A^{2} \rightarrow[0, \infty)$ such that

$$
\begin{aligned}
\|D f(x, y)\|_{B} & \leq \varphi(x, y), \\
\|f(x y)-f(x) f(y)\|_{B} & \leq \varphi(x, y)
\end{aligned}
$$

for all $x, y \in A$. If for each $x \in A$ the mapping $f(t x)$ is continuous in $t \in \mathbb{R}$ and if there exists an $L<1$ such that $\varphi(x, y) \leq 2 L \varphi\left(\frac{x}{2}, \frac{y}{2}\right)$ for all $x, y \in A$, then there exists a unique homomorphism $H: A \rightarrow B$ such that

$$
\|f(x)-H(x)\|_{B} \leq \frac{L}{1-L} \varphi(x, 0)
$$

for all $x \in A$. 
Proof. Consider the set

$$
X:=\{g: A \rightarrow B\}
$$

and introduce the generalized metric on $X$ :

$$
d(g, h)=\inf \left\{C \in \mathbb{R}_{+}:\|g(x)-h(x)\|_{B} \leq C \varphi(x, 0) \text { for all } x \in A\right\} .
$$

It is easy to show that $(X, d)$ is complete.

Now we consider the linear mapping $J: X \rightarrow X$ such that

$$
J g(x):=\frac{1}{2} g(2 x)
$$

for all $x \in A$.

By Theorem 3.1 of [3],

$$
d(J g, J h) \leq L d(g, h)
$$

for all $g, h \in X$.

Letting $y=0$ in (2.1), we get

$$
\left\|2 f\left(\frac{x}{2}\right)-f(x)\right\|_{B} \leq \varphi(x, 0)
$$

for all $x \in A$. So

$$
\left\|f(x)-\frac{1}{2} f(2 x)\right\|_{B} \leq \frac{1}{2} \varphi(2 x, 0) \leq L \varphi(x, 0)
$$

for all $x \in A$. Hence $d(f, J f) \leq L$.

By Theorem 1.3, there exists a mapping $H: A \rightarrow B$ such that

(1) $H$ is a fixed point of $J$, i.e.,

$$
H(2 x)=2 H(x)
$$

for all $x \in A$. The mapping $H$ is a unique fixed point of $J$ in the set

$$
Y=\{g \in X: d(f, g)<\infty\} .
$$

This implies that $H$ is a unique mapping satisfying (2.4) such that there exists $C \in(0, \infty)$ satisfying

$$
\|H(x)-f(x)\|_{B} \leq C \varphi(x, 0)
$$

for all $x \in A$.

(2) $d\left(J^{n} f, H\right) \rightarrow 0$ as $n \rightarrow \infty$. This implies the equality

$$
\lim _{n \rightarrow \infty} \frac{f\left(2^{n} x\right)}{2^{n}}=H(x)
$$

for all $x \in A$. 
(3) $d(f, H) \leq \frac{1}{1-L} d(f, J f)$, which implies the inequality

$$
d(f, H) \leq \frac{L}{1-L} .
$$

This implies that the inequality (2.3) holds.

One can easily show that

$$
\lim _{j \rightarrow \infty} \frac{1}{2^{j}} \varphi\left(2^{j} x, 2^{j} y\right)=0
$$

for all $x, y \in A$. It follows from (2.1), (2.5) and (2.6) that

$$
\begin{aligned}
& \left\|H\left(\frac{x+y}{2}\right)+H\left(\frac{x-y}{2}\right)-H(x)\right\|_{B} \\
& =\lim _{n \rightarrow \infty} \frac{1}{2^{n}}\left\|f\left(2^{n-1}(x+y)\right)+f\left(2^{n-1}(x-y)\right)-f\left(2^{n} x\right)\right\|_{B} \\
& \leq \lim _{n \rightarrow \infty} \frac{1}{2^{n}} \varphi\left(2^{n} x, 2^{n} y\right)=0
\end{aligned}
$$

for all $x, y \in A$. So

$$
H\left(\frac{x+y}{2}\right)+H\left(\frac{x-y}{2}\right)=H(x)
$$

for all $x, y \in A$. Letting $z=\frac{x+y}{2}$ and $w=\frac{x-y}{2}$ in the above equation, we get

$$
H(z)+H(w)=H(z+w)
$$

for all $z, w \in A$. So the mapping $H: A \rightarrow B$ is Cauchy additive, i.e., $H(z+w)=H(z)+H(w)$ for all $z, w \in A$.

By the same reasoning as in the proof of Theorem 1.1 [20], one can show that the mapping $H: A \rightarrow B$ is $\mathbb{R}$-linear.

It follows from (2.2) that

$$
\begin{aligned}
\|H(x y)-H(x) H(y)\|_{B} & =\lim _{n \rightarrow \infty} \frac{1}{4^{n}}\left\|f\left(4^{n} x y\right)-f\left(2^{n} x\right) f\left(2^{n} y\right)\right\|_{B} \\
& \leq \lim _{n \rightarrow \infty} \frac{1}{4^{n}} \varphi\left(2^{n} x, 2^{n} y\right) \leq \lim _{n \rightarrow \infty} \frac{1}{2^{n}} \varphi\left(2^{n} x, 2^{n} y\right)=0
\end{aligned}
$$

for all $x, y \in A$. So

$$
H(x y)=H(x) H(y)
$$

for all $x, y \in A$.

Thus $H: A \rightarrow B$ is a homomorphism satisfying (2.3), as desired. 
Corollary 2.2. Let $r<1$ and $\theta$ be nonnegative real numbers, and let $f: A \rightarrow B$ be a mapping such that

$$
\begin{aligned}
\|D f(x, y)\|_{B} & \leq \theta\left(\|x\|_{A}^{r}+\|y\|_{A}^{r}\right), \\
\|f(x y)-f(x) f(y)\|_{B} & \leq \theta\left(\|x\|_{A}^{r}+\|y\|_{A}^{r}\right)
\end{aligned}
$$

for all $x, y \in A$. If for each $x \in A$ the mapping $f(t x)$ is continuous in $t \in \mathbb{R}$, then there exists a unique homomorphism $H: A \rightarrow B$ such that

$$
\|f(x)-H(x)\|_{B} \leq \frac{2^{r} \theta}{2-2^{r}}\|x\|_{A}^{r}
$$

for all $x \in A$.

Proof. The proof follows from Theorem 2.1 by taking

$$
\varphi(x, y):=\theta\left(\|x\|_{A}^{r}+\|y\|_{A}^{r}\right)
$$

for all $x, y \in A$. Then we can choose $L=2^{r-1}$ and we get the desired result.

Theorem 2.3. Let $f: A \rightarrow B$ be a mapping for which there exists a function $\varphi: A^{2} \rightarrow[0, \infty)$ satisfying (2.1) and (2.2). If for each $x \in A$ the mapping $f(t x)$ is continuous in $t \in \mathbb{R}$ and if there exists an $L<1$ such that $\varphi\left(\frac{x}{2}, \frac{y}{2}\right) \leq \frac{L}{4} \varphi(x, y)$ for all $x, y \in A$, then there exists a unique homomorphism $H: A \rightarrow B$ such that

$$
\|f(x)-H(x)\|_{B} \leq \frac{1}{1-L} \varphi(x, 0)
$$

for all $x \in A$.

Proof. Consider the complete generalized metric space $(X, d)$ given in the proof of Theorem 2.1.

Now we consider the linear mapping $J: X \rightarrow X$ such that

$$
J g(x):=2 g\left(\frac{x}{2}\right)
$$

for all $x \in A$.

By Theorem 3.1 of [3],

$$
d(J g, J h) \leq L d(g, h)
$$

for all $g, h \in X$.

Letting $y=0$ in (2.1), we get

$$
\left\|2 f\left(\frac{x}{2}\right)-f(x)\right\|_{B} \leq \varphi(x, 0)
$$


for all $x \in A$. So

$$
\left\|f(x)-2 f\left(\frac{x}{2}\right)\right\|_{B} \leq \varphi(x, 0) \leq \frac{L}{2} \varphi(2 x, 0)
$$

for all $x \in A$. Hence $d(f, J f) \leq 1$.

By Theorem 1.3, there exists a mapping $H: A \rightarrow B$ such that

(1) $H$ is a fixed point of $J$. This implies that $H$ is a unique mapping satisfying (2.4) such that there exists $C \in(0, \infty)$ satisfying

$$
\|H(x)-f(x)\|_{B} \leq C \varphi(x, 0)
$$

for all $x \in A$.

(2) $d\left(J^{n} f, H\right) \rightarrow 0$ as $n \rightarrow \infty$. This implies the equality

$$
\lim _{n \rightarrow \infty} 2^{n} f\left(\frac{x}{2^{n}}\right)=H(x)
$$

for all $x \in A$.

(3) $d(f, H) \leq \frac{1}{1-L} d(f, J f)$, which implies the inequality

$$
d(f, H) \leq \frac{1}{1-L} .
$$

This implies that the inequality (2.9) holds.

One can easily show that

$$
\lim _{j \rightarrow \infty} 4^{j} \varphi\left(\frac{x}{2^{j}}, \frac{y}{2^{j}}\right)=0
$$

for all $x, y \in A$. By (2.1), we see that

$$
\begin{aligned}
& \left\|H\left(\frac{x+y}{2}\right)+H\left(\frac{x-y}{2}\right)-H(x)\right\|_{B} \\
& =\lim _{n \rightarrow \infty} 2^{n}\left\|f\left(\frac{x+y}{2^{n+1}}\right)+f\left(\frac{x-y}{2^{n+1}}\right)-f\left(\frac{x}{2^{n}}\right)\right\|_{B} \\
& \leq \lim _{n \rightarrow \infty} 2^{n} \varphi\left(\frac{x}{2^{n}}, \frac{y}{2^{n}}\right) \leq \lim _{n \rightarrow \infty} 4^{n} \varphi\left(\frac{x}{2^{n}}, \frac{y}{2^{n}}\right)=0
\end{aligned}
$$

for all $x, y \in A$.

By the proof of Theorem 2.1, the mapping $H: A \rightarrow B$ is Cauchy additive.

By the same reasoning as in the proof of Theorem 1.1 [20], one can show that the mapping $H: A \rightarrow B$ is $\mathbb{R}$-linear. 
It follows from (2.2) that

$$
\begin{aligned}
\|H(x y)-H(x) H(y)\|_{B} & =\lim _{n \rightarrow \infty} 4^{n}\left\|f\left(\frac{x y}{4^{n}}\right)-f\left(\frac{x}{2^{n}}\right) f\left(\frac{y}{2^{n}}\right)\right\|_{B} \\
& \leq \lim _{n \rightarrow \infty} 4^{n} \varphi\left(\frac{x}{2^{n}}, \frac{y}{2^{n}}\right)=0
\end{aligned}
$$

for all $x, y \in A$. So

$$
H(x y)=H(x) H(y)
$$

for all $x, y \in A$.

Thus $H: A \rightarrow B$ is a homomorphism satisfying (2.9), as desired.

Corollary 2.4. Let $r>2$ and $\theta$ be nonnegative real numbers, and let $f: A \rightarrow B$ be a mapping satisfying (2.7) and (2.8). If for each $x \in A$ the mapping $f(t x)$ is continuous in $t \in \mathbb{R}$, then there exists a unique homomorphism $H: A \rightarrow B$ such that

$$
\|f(x)-H(x)\|_{B} \leq \frac{2^{r} \theta}{2^{r}-4}\|x\|_{A}^{r}
$$

for all $x \in A$.

Proof. The proof follows from Theorem 2.3 by taking

$$
\varphi(x, y):=\theta\left(\|x\|_{A}^{r}+\|y\|_{A}^{r}\right)
$$

for all $x, y \in A$. Then we can choose $L=2^{2-r}$ and we get the desired result.

\section{Stability of derivations on Banach algebras}

Note that an $\mathbb{R}$-linear mapping $\delta: A \rightarrow A$ is called a derivation on $A$ if $\delta$ satisfies $\delta(x y)=\delta(x) y+x \delta(y)$ for all $x, y \in A$.

We prove the generalized Hyers-Ulam stability of derivations on Banach algebras for the functional equation $D f(x, y)=0$.

Theorem 3.1. Let $f: A \rightarrow A$ be a mapping for which there exists a function $\varphi: A^{2} \rightarrow[0, \infty)$ such that

$$
\begin{aligned}
\|D f(x, y)\|_{A} & \leq \varphi(x, y), \\
\|f(x y)-f(x) y-x f(y)\|_{A} & \leq \varphi(x, y)
\end{aligned}
$$


for all $x, y \in A$. If there exists an $L<1$ such that $\varphi(x, y) \leq 2 L \varphi\left(\frac{x}{2}, \frac{y}{2}\right)$ for all $x, y \in A$. If for each $x \in A$ the mapping $f(t x)$ is continuous in $t \in \mathbb{R}$, then there exists a unique derivation $\delta: A \rightarrow A$ such that

$$
\|f(x)-\delta(x)\|_{A} \leq \frac{L}{1-L} \varphi(x, 0)
$$

for all $x \in A$.

Proof. By the same reasoning as the proof of Theorem 2.1, there exists a unique $\mathbb{R}$-linear mapping $\delta: A \rightarrow A$ satisfying (3.3). The mapping $\delta: A \rightarrow A$ is given by

for all $x \in A$.

$$
\delta(x)=\lim _{n \rightarrow \infty} \frac{f\left(2^{n} x\right)}{2^{n}}
$$

It follows from (3.2) that

$$
\begin{aligned}
& \|\delta(x y)-\delta(x) y-x \delta(y)\|_{A} \\
& =\lim _{n \rightarrow \infty} \frac{1}{4^{n}}\left\|f\left(4^{n} x y\right)-f\left(2^{n} x\right) \cdot 2^{n} y-2^{n} x f\left(2^{n} y\right)\right\|_{A} \\
& \leq \lim _{n \rightarrow \infty} \frac{1}{4^{n}} \varphi\left(2^{n} x, 2^{n} y\right) \leq \lim _{n \rightarrow \infty} \frac{1}{2^{n}} \varphi\left(2^{n} x, 2^{n} y\right)=0
\end{aligned}
$$

for all $x, y \in A$. So

$$
\delta(x y)=\delta(x) y+x \delta(y)
$$

for all $x, y \in A$. Thus $\delta: A \rightarrow A$ is a derivation satisfying (3.3).

Corollary 3.2. Let $r<1$ and $\theta$ be nonnegative real numbers, and let $f: A \rightarrow A$ be a mapping such that

$$
\begin{aligned}
\|D f(x, y)\|_{A} & \leq \theta\left(\|x\|_{A}^{r}+\|y\|_{A}^{r}\right), \\
\|f(x y)-f(x) y-x f(y)\|_{A} & \leq \theta\left(\|x\|_{A}^{r}+\|y\|_{A}^{r}\right)
\end{aligned}
$$

for all $x, y \in A$. If for each $x \in A$ the mapping $f(t x)$ is continuous in $t \in \mathbb{R}$, then there exists a unique derivation $\delta: A \rightarrow A$ such that

$$
\|f(x)-\delta(x)\|_{A} \leq \frac{2^{r} \theta}{2-2^{r}}\|x\|_{A}^{r}
$$

for all $x \in A$.

Proof. The proof follows from Theorem 3.1 by taking

$$
\varphi(x, y):=\theta\left(\|x\|_{A}^{r}+\|y\|_{A}^{r}\right)
$$

for all $x, y \in A$. Then we can choose $L=2^{r-1}$ and we get the desired result. 
Theorem 3.3. Let $f: A \rightarrow A$ be a mapping for which there exists a function $\varphi: A^{2} \rightarrow[0, \infty)$ satisfying (3.1) and (3.2). If there exists an $L<1$ such that $\varphi\left(\frac{x}{2}, \frac{y}{2}\right) \leq \frac{L}{4} \varphi(x, y)$ for all $x, y \in A$. If for each $x \in A$ the mapping $f(t x)$ is continuous in $t \in \mathbb{R}$, then there exists a unique derivation $\delta: A \rightarrow A$ such that

$$
\|f(x)-\delta(x)\|_{A} \leq \frac{1}{1-L} \varphi(x, 0)
$$

for all $x \in A$.

Proof. By the same reasoning as the proof of Theorem 2.3, there exists a unique $\mathbb{R}$-linear mapping $\delta: A \rightarrow A$ satisfying (3.6). The mapping $\delta: A \rightarrow A$ is given by

$$
\delta(x)=\lim _{n \rightarrow \infty} 2^{n} f\left(\frac{x}{2^{n}}\right)
$$

for all $x \in A$.

It follows from (3.2) that

$$
\begin{aligned}
\| \delta(x y) & -\delta(x) y-x \delta(y) \|_{A} \\
= & \lim _{n \rightarrow \infty} 4^{n}\left\|f\left(\frac{x y}{4^{n}}\right)-f\left(\frac{x}{2^{n}}\right) \cdot \frac{y}{2^{n}}-\frac{x}{2^{n}} f\left(\frac{y}{2^{n}}\right)\right\|_{A} \\
\leq & \lim _{n \rightarrow \infty} 4^{n} \varphi\left(\frac{x}{2^{n}}, \frac{y}{2^{n}}\right)=0
\end{aligned}
$$

for all $x, y \in A$. So

$$
\delta(x y)=\delta(x) y+x \delta(y)
$$

for all $x, y \in A$. Thus $\delta: A \rightarrow A$ is a derivation satisfying (3.6).

Corollary 3.4. Let $r>2$ and $\theta$ be nonnegative real numbers, and let $f: A \rightarrow A$ be a mapping satisfying (3.4) and (3.5). If for each $x \in A$ the mapping $f(t x)$ is continuous in $t \in \mathbb{R}$, then there exists a unique derivation $\delta: A \rightarrow A$ such that

$$
\|f(x)-\delta(x)\|_{A} \leq \frac{2^{r} \theta}{2^{r}-4}\|x\|_{A}^{r}
$$

for all $x \in A$.

Proof. The proof follows from Theorem 3.3 by taking

$$
\varphi(x, y):=\theta\left(\|x\|_{A}^{r}+\|y\|_{A}^{r}\right)
$$

for all $x, y \in A$. Then we can choose $L=2^{2-r}$ and we get the desired result. 


\section{References}

[1] C. Baak, Cauchy-Rassias stability of Cauchy-Jensen additive mappings in Banach spaces, Acta Math. Sin. (Engl. Ser.) 22 (2006), 1789-1796.

[2] C. Baak, D. Boo and Th.M. Rassias, Generalized additive mapping in Banach modules and isomorphisms between $C^{*}$-algebras, J. Math. Anal. Appl. 314 (2006), 150-161.

[3] L. Cădariu and V. Radu, Fixed points and the stability of Jensen's functional equation, J. Inequal. Pure Appl. Math. 4, no. 1 (2003), Art. ID 4.

[4] P.W. Cholewa, Remarks on the stability of functional equations, Aequationes Math. 27 (1984), 76-86.

[5] S. Czerwik, On the stability of the quadratic mapping in normed spaces, Abh. Math. Sem. Univ. Hamburg 62 (1992), 59-64.

[6] J. Diaz and B. Margolis, A fixed point theorem of the alternative for contractions on a generalized complete metric space, Bull. Amer. Math. Soc. (N.S) 74 (1968), 305-309.

[7] P. Găvruta, A generalization of the Hyers-Ulam-Rassias stability of approximately additive mappings, J. Math. Anal. Appl. 184 (1994), 431-436.

[8] D.H. Hyers, On the stability of the linear functional equation, Proc. Natl. Acad. Sci. U.S.A. 27 (1941), 222-224.

[9] D.H. Hyers, G. Isac and Th.M. Rassias, Topics in Nonlinear Analysis and Applications, World Scientific Publishing Co., Singapore, New Jersey, London, 1997.

[10] D.H. Hyers, G. Isac and Th.M. Rassias, Stability of Functional Equations in Several Variables, Birkhäuser, Basel, 1998.

[11] F.H. Hyers and Th.M. Rassias, Approximate homomorphisms, Aequationes Math. 44 (1992), 125-153.

[12] G. Isac and Th.M. Rassias, Stability of $\psi$-additive mappings: appications to nonlinear analysis, Internat. J. Math. Math. Sci. 19 (1996), 219-228.

[13] S.-M. Jung, Hyers-Ulam-Rassias Stability of Functional Equations in Mathematical Analysis, Hadronic Press, Palm Harbor, Florida, 2001.

[14] C. Park, Fixed points and Hyers-Ulam-Rassias stability of Cauchy-Jensen functional equations in Banach algebras, Fixed Point Theory Appl. 2007 (2007), Art. ID 50175.

[15] C. Park and J. Hou, Homomorphisms between $C^{*}$-algebras associated with the Trif functional equation and linear derivations on $C^{*}$-algebras, J. Korean Math. Soc. 41 (2004), 461-477.

[16] C. Park, J. Hou and S. Oh, Homomorphisms between $\mathrm{JC}^{*}$-algebras and between Lie $C^{*}$-algebras, Acta Math. Sin. (Engl.Ser.) 21 (2005), 1391-1398.

[17] C. Park and Th.M. Rassias, On a generalized Trif's mapping in Banach modules over a $C^{*}$-algebra, J. Korean Math. Soc. 43 (2006), 323-356.

[18] J.M. Rassias, On approximation of approximately linear mappings by linear mappings, Bull. Sci. Math. 108 (1984), 445-446.

[19] J.M. Rassias, Solution of a problem of Ulam, J. Approx. Theory 57 (1989), 268-273. 
[20] Th.M. Rassias, On the stability of the linear mapping in Banach spaces, Proc. Amer. Math. Soc. 72 (1978), 297-300.

[21] Th.M. Rassias, On modified Hyers-Ulam sequence, J. Math. Anal. Appl. 158 (1991), 106-113.

[22] Th.M. Rassias, On the stability of the quadratic functional equation and its applications, Studia Univ. Babeş-Bolyai, Ser. Math. XLIII (1998), 89-124.

[23] Th.M. Rassias, The problem of S.M. Ulam for approximately multiplicative mappings, J. Math. Anal. Appl. 246 (2000), 352-378.

[24] Th.M. Rassias, On the stability of functional equations in Banach spaces, J. Math. Anal. Appl. 251 (2000), 264-284.

[25] Th.M. Rassias, On the stability of functional equations and a problem of Ulam, Acta Appl. Math. 62 (2000), 23-130.

[26] Th.M. Rassias and P. Šemrl, On the behavior of mappings which do not satisfy Hyers-Ulam stability, Proc. Amer. Math. Soc. 114 (1992), 989-993.

[27] Th.M. Rassias and P. Šemrl, On the Hyers-Ulam stability of linear mappings, J. Math. Anal. Appl. 173 (1993), 325-338.

[28] Th.M. Rassias and K. Shibata, Variational problem of some quadratic functionals in complex analysis, J. Math. Anal. Appl. 228 (1998), 234-253.

[29] F. Skof, Proprietà locali e approssimazione di operatori, Rend. Sem. Mat. Fis. Milano 53 (1983), 113-129.

[30] S.M. Ulam, Problems in Modern Mathematics, Wiley, New York, 1960.

Department of Mathematics

Hanyang University

Seoul 133-791, Republic of Korea

E-mail: baak@hanyang.ac.kr

Department of Mathematics Education

Mokwon University

Daejeon 302-729, Republic of Korea

E-mail: wgpark@mokwon.ac.kr

Department of Mathematics

Daejin University

Kyeonggi 487-711, Republic of Korea

E-mail: jrlee@daejin.ac.kr

Department of Mathematics

National Technical University of Athens

Zografou Campus, 15780 Athens, Greece

E-mail: trassias@math.ntua.gr 